\title{
Importance of sustainable polymers for modern society and development
}

\author{
Md. Rezaur Rahman, Nur-Azzah Afifah Binti Taib, Muhammad Khusairy \\ Bin Bakri and Siti Noor Linda Taib \\ Faculty of Engineering, Universiti Malaysia Sarawak, Jalan Datuk Mohammad Musa, \\ Kota Samarahan, Malaysia
}

\subsection{Introduction}

\subsubsection{Polymer}

Polymers are an essential material that is widely used in a lot of daily life's products and good, and it is unimaginable to live without it. Polymers were formed when many small molecules named monomers were linked together to form long chains. People have been using polymers for many years in their lives, but they did not know it well until the Second World War. This is due to relatively few materials that were available for people for a civilized life. Materials such as steel, glass, wood, and brick were used for construction, while agricultural products such as cotton and jute were used for clothing or fabric manufacture. The introduction of this new material, that is, polymers, was due to the rapid increase in demand for manufactured products. It is safe to say that polymer-based products are all around us, from the synthetic fibers that make our clothes to Teflon-coated cookware. The lists are nearly endless (Namazi, 2017).

Derived from classical Greek words "poly" (means "many") and "meres" (means "parts") makes up the word "polymer" or sometimes "macromolecule," and this modern view of polymers was introduced in the 1920 s by the German chemist, Hermann Staudinger (Namazi, 2017; Rasmussen, 2018). Typically, the molecular weight of the polymer molecule is high, which is between 10,000 and 1,000,000 g/mol and consisted of several structural units that are bound together by covalent bonds. Next, via chemical reaction of monomers, the polymers would be obtained. This is because in suitable condition, the formation of polymer chain can be obtained as the monomers can react with another molecule, either from the same or different type. The formation of natural polymers occurred when this reaction process happened naturally, while the synthetic polymers are when it was man-made (Namazi, 2017). At an early date, natural polymers were chemically modified in an empirical manner. It was then followed by the production of completely synthetic substances that we now understand to be polymers in the 19th century. The polymers produced include polyaniline 